\title{
OSCILLATION OF SUPERLINEAR MATRIX DIFFERENTIAL EQUATIONS
}

\author{
CALVIN D. AHLBRANDT, JERRY RIDENHOUR AND RUSSELL C. THOMPSON
}

(Communicated by Kenneth R. Meyer)

\begin{abstract}
The main theorems extend to matrix differential equations, Atkinson's classic theorem giving necessary and sufficient conditions for the oscillation of superlinear second-order scalar differential equations. The theorems improve recent results of Kura and of Butler and Erbe by removing a very restrictive hypothesis that solutions be symmetric.
\end{abstract}

\section{INTRODUCTION}

In his classic paper [4], Atkinson showed that when $f(t)$ is positive and continuous for $t$ in $[0, \infty)$, a necessary and sufficient condition for the secondorder superlinear scalar equation

$$
y^{\prime \prime}+f(t) y^{2 n+1}=0
$$

to be oscillatory is that $\int_{0}^{\infty} t f(t) d t=\infty$. The papers of Kura [18] and Butler and Erbe [6] extend Atkinson's theorem to the case of superlinear matrix equations; however, their theorems apply only to symmetric matrix solutions. This symmetry assumption is very restrictive as noted at the end of $\S 2$. The main results of this paper also extend Atkinson's theorem to superlinear matrix equations, with the principal advantage of our theorems being that they apply to solutions which may or may not be symmetric.

Atkinson's paper and the corresponding work of Belohorec [5] on sublinear equations provided the impetus for a very large body of research on the oscillation theory of nonlinear scalar differential equations. The survey article by Kartsatos [14] contains over 300 such references, some of which are themselves survey articles containing additional references. In the past ten years, the oscillation theory for systems of ordinary differential equations, both linear and nonlinear, has received considerable attention. To give the reader a brief and very incomplete sampling of the research in these directions, we cite $[15,17$,

Received by the editors October 2, 1987.

1980 Mathematics Subject Classification (1985 Revision). Primary 34C10.

Theorem 1 was presented by Jerry Ridenhour at the Eigth Annual Midwest Differential Equations Conference held October 18-19, 1979, at Oklahoma State University. 
18,20 , and 22] as indicative of the work done on nonlinear systems and [1-3, $7,8,10,12,16,19$, and 21] as examples of the work on linear systems.

\section{Statement of Results}

In passing from the scalar equation (1) to a superlinear matrix differential equation, there are many ways to write the superlinear matrix term corresponding to the term $f(t) y^{2 n+1}$ in (1). Due to the noncommutativity of matrix multiplication, the analysis (as we shall see below) may vary depending on the way in which this nonlinear term is expressed. To begin, we suppose throughout that $F(t)$ is a continuous $m \times m$ complex matrix-valued function on $[0, \infty)$ such that $F(t)$ is Hermitian and positive definite for each $t$ in $[0, \infty)$. We introduce an equation with a cubic nonlinearity in two forms, namely

$$
Y^{\prime \prime}+\left(Y F(t) Y^{*}\right) Y=0
$$

and

$$
Y^{\prime \prime}+\left(Y^{*} F(t) Y\right) Y=0 .
$$

Here, as elsewhere, $Y^{*}$ denotes the adjoint (i.e., the conjugate transpose) of $Y$.

For a positive integer $n$, let $H_{n}$, the general matrix analogue of the scalar power $y^{n}$, represent any product of the form

$$
H_{n}=H_{n}(Y)=Z_{1} Z_{2} \cdots Z_{n},
$$

where, for each $i=1,2, \ldots, n$, either $Z_{i}=Y$ or $Z_{i}=Y^{*}$. To unify notation, we let $H_{0}$ be the $m \times m$ identity matrix. The matrix equations analogous to (1) are then

$$
Y^{\prime \prime}+\left(Y H_{n} F(t) H_{n}^{*} Y^{*}\right) Y=0
$$

and

$$
Y^{\prime \prime}+\left(Y^{*} H_{n} F(t) H_{n}^{*} Y\right) Y=0 .
$$

Although (2) and (3) are special cases (with $n=0$ ) of (4) and (5), they are considered separately since the proofs amount to first establishing results for (2) and (3) and then extending to (4) and (5). It will be important that the expressions in parentheses in (4) and (5) are Hermitian; however, these expressions could be commuted with $Y$ in the nonlinear term, and analogous results would follow.

As noted above, we are considering $F(t)$ to be complex matrix-valued; consequently, we consider the complex matrix-valued solutions of (4) (or (5)). Since most of the results in the literature are stated for the real case, we remark that our results remain true (with the same proofs) when the adjoint of a matrix is replaced by the transpose, Hermitian matrices are replaced by symmetric matrices, and complex matrix-valued solutions are replaced by real matrix-valued solutions. 
We consider only those $m \times m$ complex matrix-valued solutions $Y(t)$ of (4) (or (5)) that extend to infinity (i.e., exist for all $t$ in some ray of the form $[a, \infty), a \geq 0)$. The problem of determining when solutions extend to infinity is nontrivial. In the appendix to [13], Hastings gives an example of a function $f(t)$ which is positive and continuous on the interval $[0, \infty)$ and such that $(1)$ with $n=1$ has at least one solution $y(t)$ defined at $t=0$ but not extensible to the interval $[0, \infty)$. However, under rather modest conditions on $f(t)$, Hastings shows that all solutions of (1) do extend to infinity; in particular, if $f(t)$ is Lipschitz continuous or monotone in some interval to the left of every positive point, Lemma 3 of [13] implies that any solution of (1) defined at $t=0$ can be extended to $[0, \infty)$. We do not pursue the problem of determining conditions on $F(t)$ so that (4) and (5) will have solutions extending to infinity, but we suspect that rather mild conditions suffice.

Following the lead of others (e.g., [17, 20, 22]), we call a solution of (4) (or (5)) prepared if $Y^{*}(t) Y^{\prime}(t)-Y^{*}(t) Y(t)$ is the zero matrix for all $t$. Differentiation shows that, for any solution $Y(t)$ of one of equations (4) or (5), the matrix $Y^{*}(t) Y^{\prime}(t)-Y^{\prime *}(t) Y(t)$ is necessarily constant; hence, $Y(t)$ is prepared when $Y^{*}\left(t_{0}\right) Y^{\prime}\left(t_{0}\right)-Y^{\prime *}\left(t_{0}\right) Y\left(t_{0}\right)=0$ for some $t_{0}$. By a zero of a matrix solution $Y$, we mean a point $t$ where the $m \times m$ matrix $Y(t)$ is singular. A matrix solution is called oscillatory if it extends to infinity and has arbitrarily large zeros. Equation (4) (or (5)) is said to be oscillatory if all prepared solutions that extend to infinity are oscillatory.

It is well known that, when allowing complex solutions, the preparedness assumption is necessary in order to avoid a mixture of oscillatory and nonoscillatory solutions, even in the case of linear scalar differential equations. For example, $e^{i t}$ is a nonprepared nonoscillatory solution of $y^{\prime \prime}+y=0$. Noussair and Swanson [20] have produced an example showing the same is true for matrix differential equations, even when restricting to real matrix-valued solutions.

We denote the maximum and minimum eigenvalues of a Hermitian matrix $H$ by $\lambda_{\max }(H)$ and $\lambda_{\min }(H)$, respectively. Our main result follows.

Theorem 1. Suppose $F(t)$ is a continuous $m \times m$ complex matrix-valued function on $[0, \infty)$ such that $F(t)$ is Hermitian and positive definite for each $t$ in $[0, \infty)$. Then (4) is oscillatory provided that

$$
\int_{0}^{\infty} t \lambda_{\max }(F(t)) d t=\infty
$$

holds, and (5) is oscillatory provided that

$$
\int_{0}^{\infty} t \lambda_{\min }(F(t)) d t=\infty
$$

holds.

We conjecture that (5) is also oscillatory with the weaker hypothesis (6) replacing (7); however, the proof that we give below does not yield this result. We have undertaken a numerical study of this question in which, for the same 
$F(t)$, solutions of (2) and solutions of (3) satisfying the same initial conditions at some point $t_{0}$ were compared to each other. In all examples, solutions of (3) either oscillated significantly more quickly or oscillated as quickly as did solutions of (2). These examples seem to indicate that (3) is also oscillatory with the weaker assumption. Of course, in the scalar case, both (6) and (7) reduce to Atkinson's condition.

Theorem 1 provides sufficient conditions for (4) and (5) to be oscillatory. As noted in a remark of Kura (see p. 225 of [18]), the fact that (6) is a necessary condition for oscillation follows easily from a result of Kartsatos and Walters [15]. We summarize that fact in a theorem.

Theorem 2. If $F(t)$ is a continuous $m \times m$ complex matrix-valued function on $[0, \infty)$ which is also Hermitian and positive definite for each $t$ in $[0, \infty)$, then (6) is a necessary condition for (4) and (5) to be oscillatory.

Combining Theorems 1 and 2, we see that (6) is both necessary and sufficient for (4) to be oscillatory.

We remark on the consequences of the assumption in [6 and 18] that the solutions $Y(t)$ are both symmetric and prepared. For symmetric solutions, (4) and (5) both reduce to

$$
Y^{\prime \prime}+Y^{n} F(t) Y^{n+1}=0 .
$$

At any point where a symmetric solution $Y(t)$ is nonsingular, it follows from taking transposes in (8) and multiplying appropriately by $Y^{-1}(t)$ that $F(t)$ commutes with $Y(t)$. The additional assumption that $Y(t)$ is prepared implies that $Y(t)$ commutes with $Y^{\prime}(t)$. Dieudonne [9] has shown that continuously differentiable matrices which commute with their derivatives must be functionally commutative (i.e., $Y(t) Y(s)=Y(s) Y(t)$ for all $t$ and $s$ ). Furthermore, as proved by Goff [11], analytic Hermitian matrices which commute with their derivatives must be functionally commutative. Hence, at points where a solution $Y(t)$ satisfies $\operatorname{det}(Y(t)) \neq 0$, the assumption that $Y(t)$ is both symmetric and prepared implies that $Y(t)$ commutes both with $F(t)$ and with $Y^{\prime}(t)$. This is clearly a very restrictive assumption. On the other hand, our results apply when $Y(t)$ is any prepared solution, a condition that can be guaranteed by correct assignment of initial conditions at a single point $t_{0}$.

\section{Proofs of THE THEOREMS}

As a tool in the proof of Theorem 1, we will need the following lemma from matrix theory.

Lemma 1. If $P$ is a positive definite $m \times m$ Hermitian matrix and $Q$ is any $m \times m$ matrix, then the following inequalities hold:

$$
\begin{aligned}
& \lambda_{\text {max }}\left(Q^{*} P Q\right) \geq \lambda_{\text {max }}\left(Q^{*} Q\right) \lambda_{\text {min }}(P), \\
& \lambda_{\text {max }}\left(Q^{*} P Q\right) \geq \lambda_{\text {min }}\left(Q^{*} Q\right) \lambda_{\text {max }}(P),
\end{aligned}
$$


and

$$
\lambda_{\text {min }}\left(Q^{*} P Q\right) \geq \lambda_{\min }\left(Q^{*} Q\right) \lambda_{\min }(P)
$$

Proof. These inequalities follow from Rayleigh's principle. The least direct is (10) so we prove (10) and leave the proofs of (9) and (11) to the reader. Let $\|\cdot\|$ denote the Euclidean norm and let $\langle\cdot, \cdot\rangle$ denote the inner product in $\mathbf{R}^{n}$. If $Q$ is singular, then (10) follows immediately since the right-hand side is then zero; hence, we assume $Q$ is nonsingular.

Choose a vector $y$ with $\|y\|=1$ so that $\langle P y, y\rangle=\lambda_{\max }(P)$. Since $Q$ is nonsingular, we choose $x$ with $Q x=y$. Then

$$
\begin{aligned}
\lambda_{\max }\left(Q^{*} P Q\right) & \geq \frac{\left\langle Q^{*} P Q x, x\right\rangle}{\langle x, x\rangle}=\frac{\langle P Q x, Q x\rangle}{\langle x, x\rangle} \\
& =\frac{\lambda_{\max }(P)}{\langle x, x\rangle}=\lambda_{\max }(P) \frac{\langle Q x, Q x\rangle}{\langle x, x\rangle} \\
& =\lambda_{\max }(P) \frac{\left\langle Q^{*} Q x, x\right\rangle}{\langle x, x\rangle} \geq \lambda_{\max }(P) \lambda_{\min }\left(Q^{*} Q\right)
\end{aligned}
$$

This completes the proof.

Proof of Theorem 1. As a first step, we show that (2) is oscillatory provided that (6) holds. Suppose to the contrary that (6) holds but there is a prepared solution $Y$ of $(2)$ that is nonsingular on some interval $[a, \infty)$ where $a \geq 0$. We make the Riccati substitution

$$
R(t)=Y^{\prime}(t) Y^{-1}(t), \quad t \in[a, \infty) .
$$

Then $R(t)$ defined by (12) is Hermitian on $[a, \infty)$ since $Y(t)$ is prepared. We now show that $R(t)$ is eventually positive definite. Differentiating and using (2), we see that

$$
R^{\prime}=-Y F Y^{*}-R^{2}
$$

Since $R^{\prime}$ is negative, $R$ is eventually nonsingular so we assume

$$
\left(R^{-1}\right)^{\prime}=R^{-1} Y F Y^{*} R^{-1}+I
$$

is valid on $[a, \infty)$. Hence, all eigenvalues of $R^{-1}(t)$ tend to infinity as $t \rightarrow \infty$, and $R(t)$ is eventually positive definite as claimed.

Solving for $t F(t)$ in (2) yields

$$
t F(t)=-t Y^{-1} Y^{\prime \prime} Y^{-1} Y^{*-1}, \quad t \in[a, \infty),
$$

where in (13), as below, the argument $t$ has been suppressed in several places to shorten the notation. We integrate both sides of (13) using integration by parts on the right-hand side and the preparedness of $Y$ to obtain

$$
\begin{array}{rl}
\int_{a}^{t} x & F(x) d x=-\left.x Y^{-1} Y^{\prime} Y^{-1} Y^{*-1}\right|_{a} ^{t} \\
& +\int_{a}^{t} Y^{-1} Y^{\prime} Y^{-1} Y^{*-1} d x-3 \int_{a}^{t} x Y^{-1} R^{2} Y^{*-1} d x
\end{array}
$$


Since $-\frac{1}{2} Y^{-1} Y^{*-1}$ is an antiderivative of $Y^{-1} Y^{\prime} Y^{-1} Y^{*-1}$, one can rearrange (14) to find that

$$
t Y^{-1} R Y^{*-1}=-\frac{1}{2}\left(Y^{*} Y\right)^{-1}-3 \int_{a}^{t} x Y^{-1} R^{2} Y^{*-1} d x-\int_{a}^{t} x F(x) d x+K
$$

for some constant Hermitian matrix $K$.

As noted on p. 1428 of [22] by Tomastik, when $A(t)$ is positive semidefinite, the conditions

$$
\lambda_{\max }\left[\int_{a}^{\infty} A(s) d s\right]=\infty \text { and } \int_{a}^{\infty} \lambda_{\max }[A(s)] d s=\infty
$$

are equivalent. Using this fact and (6), we see that the minimum eigenvalue of the right-hand side of (15) approaches $-\infty$ as $t \rightarrow \infty$. This contradicts $R(t)$ being eventually positive definite and completes this part of the proof.

As a second step in the proof of Theorem 1, we show that (3) is oscillatory provided that (7) holds. Suppose not. Let (7) hold and let $Y(t)$ be a prepared solution of (3) that is nonsingular on $[a, \infty)$. We again let $R(t)=Y^{\prime}(t) Y^{-1}(t)$ for $t \geq a$ and use the same argument as above to show that $R(t)$ is eventually positive definite. However, the remainder of the proof is different since isolating $t F(t)$ and integrating by parts does not easily lead to a nice analogue of equation (15). Alternatively, we solve equation (3) for $t Y^{-1} Y^{\prime \prime} Y^{-1} Y^{*-1}$ which results in

$$
t Y^{-1} Y^{\prime \prime} Y^{-1} Y^{*-1}=-t Y^{-1} Y^{*} F(t) Y Y^{*-1},
$$

valid for $t \in[a, \infty)$. We integrate (16) to obtain

$$
\begin{gathered}
t Y^{-1} R Y^{*-1}=-\frac{1}{2}\left(Y^{*} Y\right)^{-1}-3 \int_{a}^{t} x Y^{-1} R^{2} Y^{*-1} d x \\
-\int_{a}^{t} x Y^{-1} Y^{*} F(x) Y Y^{*-1} d x+K
\end{gathered}
$$

as the analogue to (15) above. From (9), it follows that

$$
\begin{aligned}
\lambda_{\max }\left(Y^{-1} Y^{*} F(x) Y Y^{*-1}\right) & \geq \lambda_{\max }\left(Y^{-1} Y^{*} Y Y^{*-1}\right) \lambda_{\min }(F(x)) \\
& \geq \lambda_{\max }\left(Y^{-1} Y^{*-1}\right) \lambda_{\min }\left(Y^{*} Y\right) \lambda_{\min }(F(x)) \\
& \geq \lambda_{\min }(F(x)) .
\end{aligned}
$$

Hence, letting $t \rightarrow \infty$, we see that (7) and (17) contradict that $R(t)$ is eventually positive definite. This completes the proof that $(3)$ is oscillatory when (7) holds.

Suppose again that (6) holds. We now use the fact that (2) is oscillatory to prove that (4) is also oscillatory. Suppose not and let $Y_{0}$ be a prepared solution of (4) that is nonsingular on $[a, \infty)$. Again let $R(t)=Y_{0}^{\prime}(t) Y_{0}^{-1}(t)$ for $t \geq a$. The same calculations as before show that $R(t)$ is eventually positive definite. Also, since $Y_{0}$ is prepared,

$$
\left(Y_{0}^{*} Y_{0}\right)^{\prime}=2 Y_{0}^{*} Y_{0}^{\prime}=2 Y_{0}^{*} Y_{0}^{\prime} Y_{0}^{-1} Y_{0}=2 Y_{0}^{*} R Y_{0}
$$


Hence, the eigenvalues of $Y_{0}^{*} Y_{0}$ are eventually increasing and therefore eventually bounded below by a positive constant. Moreover, $Y^{*} Y$ and $Y Y^{*}$ have the same eigenvalues.

Let $F_{0}(t)=H_{n}\left(Y_{0}\right) F(t) H_{n}^{*}\left(Y_{0}\right)$. Then the equation

$$
Y^{\prime \prime}+\left(Y F_{0}(t) Y^{*}\right) Y=0
$$

has $Y_{0}$ as a prepared and eventually nonsingular solution. By (10) and (11), we see

$$
\begin{aligned}
\lambda_{\text {max }}\left(F_{0}(t)\right) & \geq \lambda_{\text {min }}\left(H_{n} H_{n}^{*}\right) \lambda_{\text {max }}(F(t)) \\
& \geq\left(\lambda_{\text {min }}\left(Y_{0} Y_{0}^{*}\right)\right)^{n} \lambda_{\text {max }}(F(t)) .
\end{aligned}
$$

Since the eigenvalues of $Y_{0} Y_{0}^{*}$ are eventually bounded below by a positive constant, it follows that

$$
\int_{0}^{\infty} t \lambda_{\max }\left(F_{0}(t)\right) d t=\infty
$$

But this contradicts what we have already proved since (18) is an equation of the same form as (2) for which (6) holds and yet there is an eventually nonsingular prepared solution.

The proof that (5) is oscillatory when (7) holds follows in similar fashion. This proves Theorem 1.

Proof of Theorem 2. To show that (6) is a necessary condition for (4) to be oscillatory, we assume

$$
\int_{0}^{\infty} t \lambda_{\max }(F(t)) d t<\infty
$$

and prove the existence of a (nonoscillatory) prepared solution $Y(t)$ of (4) that converges to $I$, the $m \times m$ identity matrix, as $t \rightarrow \infty$. As noted by Kura [18], this follows easily from Theorem 1 of [15]. To match the notation in [15], let

$$
\begin{gathered}
P(t, X)=X H_{n}(X) F(t) H_{n}^{*}(X) X^{*} X, \\
Q(t) \equiv 0, \quad V(t) \equiv 0,
\end{gathered}
$$

and

$$
Q(t,\|Y\|)=\lambda_{\max }(F(t))\|Y\|^{2 n+1} .
$$

Since (19) holds, Theorem 1 of [15] applies directly with $\lambda=1$ to show that (4) has a solution $Y(t)$ with $\lim _{t \rightarrow \infty} Y(t)=I$. Also, the proof of Theorem 1 in [15] gives that the derivative of the solution $Y(t)$ satisfies

$$
\left\|Y^{\prime}(t)\right\| \leq \int_{t}^{\infty} s \lambda_{\max }(F(s)) d s,
$$

where $\left\|Y^{\prime}(t)\right\|$ denotes the matrix norm induced by the Euclidean vector norm. Hence, it follows that

$$
\lim _{t \rightarrow \infty}\left[Y^{*}(t) Y^{\prime}(t)-Y^{\prime *}(t) Y(t)\right]=0
$$

and $Y(t)$ is prepared.

This completes the proof in the case of (4). The proof that condition (6) is necessary for (5) to be oscillatory follows in the same way. 


\section{REFERENCES}

1. S. Ahmad, On Sturmian theory for second order systems, Proc. Amer. Math. Soc. 87 (1983), 661-665.

2. S. Ahmad and A. C. Lazer, An n-dimensional extension of the Sturm separation and comparison theory to a class of nonselfadjoint systems, SIAM J. Math. Anal. 6 (1978), 1137-1150.

3. S. Ahmad and C. C. Travis, Oscillation criteria for second-order differential systems, Proc. Amer. Math. Soc. 71 (1978), 247-252.

4. F. V. Atkinson, On second-order non-linear oscillations, Pacific J. Math. 5 (1955), 643-647.

5. S. Belohorec, Oscillatory solutions of certain non-linear differential equations of second order, Mat.-Fyz. Casopis Sloven. Akad. Vied. 11 (1961), 250-255.

6. G. J. Butler and L. H. Erbe, Oscillation theory for second order differential systems with functional commutative coefficients, Differential and Integral Equations (Proc. Conf. Twelfth and Thirteenth Midwest; J. L. Henderson, ed.) Institute of Applied Mathematics, University of Missouri-Rolla, 1985, pp. 15-18.

7. G. J. Butler, L. H. Erbe and A. B. Mingarelli, Riccati techniques and variational principles in oscillation theory for linear systems, Trans. Amer. Math. Soc. 302 (1987), 263-282.

8. R. Byers, B. J. Harris and M. K. Kwong, Weighted means and oscillation conditions for second order matrix differential equations, J. Differential Equations 61 (1986), 164-177.

9. J. Dieudonné, Sur un Theorèm de Schwerdtfeger, Ann. Polon. Math. 24 (1974), 87-88.

10. G. T. Etgen and J. F. Pawlowski, A comparison theorem and oscillation criteria for second order differential systems, Pacific J. Math. 72 (1977), 59-69.

11. S. Goff, Hermitian function matrices which commute with their derivative, Linear Algebra and Appl. 36 (1981), 33-40.

12. P. Hartman, Oscillation criteria for self-adjoint second-order differential systems and "principal sectional curvatures", J. Differential Equations 34 (1979), 326-338.

13. S. P. Hastings, Boundary value problems in one differential equation with a discontinuity, J. Differential Equations 1 (1965), 346-369.

14. A. G. Kartsatos, Recent results on oscillation of solutions of forced and perturbed nonlinear differential equations of even order, Stability of Dynamical Systems, Theory and Applications (John R. Graef, ed.) Marcel Dekker, New York, 1977, pp. 17-72.

15. A. G. Kartsatos and T. Walters, Some oscillation results for matrix and vector differential equations with forcing term, J. Math. Anal. Appl. 73 (1980), 506-513.

16. M. S. Keener and C. C. Travis, Sturmian theory for a class of nonselfadjoint differential systems, Ann. Mat. Pura Appl. 123 (1980), 247-266.

17. K. Kreith, Oscillation criteria for nonlinear matrix differential equations, Proc. Amer. Math. Soc. 26 (1970), 270-272.

18. T. Kura, A matrix analogue of Atkinson's oscillation theorem, Funkcialaj Ekvacioj 25 (1982), 223-226.

19. M. K. Kwong and H. G. Kaper, Oscillation of two-dimensional linear second order differential systems, J. Differential Equations 56 (1985), 195-205.

20. E. S. Noussair and C. A. Swanson, Oscillation criteria for differential systems, J. Math. Anal. Appl. 36 (1971), 575-580.

21. K. Schmitt and H. L. Smith, Positive solutions and conjugate points for systems of differential equations, Nonlinear Anal. 2 (1978), 93-105.

22. E. C. Tomastik, Oscillation of nonlinear matrix differential equations of second order, Proc. Amer. Math. Soc. 19 (1968), 1427-1431.

Department of Mathematics, University of Missouri-Columbia, Columbia, Missouri 65201 (Calvin D. Ahlbrandt)

Department of Mathematics Utah State University, Logan, Utah 84322 (Jerry Ridenhour and Russell C. Thompson) 